\title{
Cytochromes and Psychotropic Drug Interactions
}

\author{
DAVID TAYLOR and MALCOLM LADER
}

The cytochrome p450 enzymes in the liver were discovered about $\mathbf{4 0}$ years ago. Soon after, it was found that these enzymes could be induced by various drugs whose metabolism would then be accelerated. Over the years, more and more subtypes of cytochromes have been characterised. A recent listing contained over 30 (Guengerich, 1992). Of these, a few appear to be of particular importance in the metabolism of commonly-used psychotropic drugs (von Moltke et al, 1994). Furthermore, predictions can now be made concerning potentially troublesome drug interactions (Murray, 1992), especially those involving antidepressants.

\section{p450-2D6}

This cytochrome is implicated in the metabolism of the antidepressants desipramine, nortriptyline, imipramine, amitriptyline and clomipramine, and the antipsychotic drugs thioridazine, fluphenazine, perphenazine and clozapine (Fischer et al, 1992). Many non-psychotic drugs are also metabolised mainly via 2D6 mechanisms including betablockers, analgesics, antiarrhythmic agents and antitussives. This enzyme is subject to genetic polymorphism, with a minority of the population (5-10\%) being slow metabolisers (Cholerton et al, 1992). These people are at risk of developing high, potentially toxic concentrations of various drugs and of suffering hazardous drug interactions. Slow metabolisers can be identified by the administration of a test compound such as debrisoquine or dextromethorphan, followed by estimation of parent to metabolite ratio in the urine. In normal metabolisers, metabolite predominates; in slow metabolisers, the parent compound persists. However, the parent compound may then be metabolised along other metabolic routes and may result in toxicity (Watkins, 1990).

The 2D6 system is susceptible to inhibition by some drugs, quinidine being the prime example. This can result in the slow metabolism of other drugs in normal metabolisers but has little effect in slow metabolisers because the liver is already deficient in this function.
Interest has recently increased in the 2D6 system because of the demonstration that some (but not all) of the selective serotonin reuptake inhibitor (SSRI) antidepressants can inhibit this enzyme in vitro. The most powerful was paroxetine $\left(\mathrm{K}_{\mathrm{i}}=0.15 \mu \mathrm{M}\right)$. Citalopram and fluvoxamine were weak inhibitors as were clomipramine, desipramine and amitriptyline (Crewe et al, 1992). Thus, paroxetine, fluoxetine and sertraline might be involved in interactions with other drugs metabolised by the 2D6 cytochrome system.

Enough is now known of the molecular configuration of the active site of the $2 \mathrm{D} 6$ protein to allow computer modelling. This is not possible yet with most other cytochromes.

\section{p450-3A family}

This comprises mainly two closely related cytochromes, $3 \mathrm{~A} 3$ and $3 \mathrm{~A} 4$. The psychotropic drugs mainly metabolised by this group include midazolam (Gorski et al, 1994), triazolam and alprazolam (von Moltke et al, 1993), and it is involved in the demethylation of imipramine, amitriptyline and clomipramine to desipramine, nortriptyline and desclomipramine, respectively. These enzymes do not show genetic polymorphism and are found in the intestinal mucosa as well as the liver. Other drugs metabolised via this system include nifedipine, quinidine, terfenadine and cyclosporin. Ketoconazole, an antifungal agent, is a powerful inhibitor of the $3 A$ enzymes. The SSRIs also have some effect but much less than on the 2D6 system.

\section{p450-1A2}

This cytochrome is also involved in the demethylation of imipramine, and in the metabolism of caffeine (Slaughter \& Edwards, 1995) and theophylline (Rasmussen et al, 1995). It is induced by smoking. Fluvoxamine is a very potent inhibitor of this system $\left(\mathrm{K}_{\mathrm{i}}\right.$ ranging from $\left.0.12-0.24 \mu \mathrm{M}\right)$ (Brøsen et al, 1993). Paroxetine is a weak inhibitor, citalopram and fluoxetine have almost no effect. Propranolol, clomipramine and amitriptyline accumulate markedly when given concomitantly with 
fluvoxamine suggesting that these drugs are also metabolised by the $1 \mathrm{~A} 2$ system.

\section{In vitro techniques}

Extracts from human liver can yield the drug metabolising fraction by ultracentrifugation and can be used to assess biotransformations of various drugs (e.g. Gascon \& Dayer, 1991). The addition of selective chemical inhibitors can help elucidate the specific cytochrome systems involved. Thus, inhibition of a reaction by quinidine suggests the participation of the 2D6 system, by ketoconazole of the $3 \mathrm{~A}$ system. Another technique is to neutralise the cytochrome protein using specific antisera, and to examine the effect on the metabolic reaction of interest (Kronbach et al, 1989). Finally, pure human cytochromes can be produced using bacterial or yeast expression systems; already about 40 genes coding various cytochromes have been identified.

\section{Practical implications}

All of the currently available SSRIs (citalopram, fluoxetine, fluvoxamine, paroxetine and sertraline) have been implicated in adverse drug interactions thought to involve cytochrome p450-2D6. Most of these relate to the elevation of plasma concentrations of tricyclic antidepressants and the occasionally serious adverse effects which result (e.g. seizures (Preskorn et al, 1990)). Fluoxetine is most frequently implicated. There are numerous case reports of interactions in the literature and at least one prospective study describing a four-fold increase in plasma desipramine concentrations (Preskorn et al, 1994). Information on other SSRIs is much more limited. Paroxetine appears to elevate desipramine levels to a similar extent as that seen with fluoxetine (Alderman et $a l, 1994)$ and fluvoxamine has been reported to increase substantially serum levels of a number of tricyclic compounds (Hartter et al, 1993; Maskall \& Lam, 1993), although this may, at least in part, be due to inhibition of other p450 enzymes. Sertraline may have a less profound effect on 2D6 (Alderman et al, 1994) but one case report described a $250 \%$ increase in desipramine levels when sertraline was given concurrently (Barros \& Asnis, 1993). Based on in vitro studies, citalopram may be the least likely of the SSRIs to give rise to adverse drug interactions caused by inhibition of 2D6. However, citalopram has been shown to prolong to a small extent the plasma elimination half life of desipramine (Gram et al, 1993).
These reports of adverse interactions have two important clinical consequences. Firstly, SSRIs and tricyclic antidepressants should probably not be given concurrently unless facilities for plasma level monitoring of tricyclics are available. Patients should also be carefully monitored for tricyclicrelated adverse effects. Secondly, transferring patients from a tricyclic to an SSRI should be undertaken with some caution. The tricyclic should generally be withdrawn slowly over a period of weeks and the SSRI introduced towards the end of the decremental schedule. Problems may also occur when starting a tricyclic after withdrawing fluoxetine (Hahn \& Griffin, 1991) which has a much longer duration of action than the other SSRIs. After withdrawing fluoxetine, the tricyclic should be introduced at a low dose (10-25 mg) and increased very slowly according to patient tolerance.

Inhibition of cytochrome p450-2D6 by SSRIs may also provoke interactions with other drugs. As already mentioned, several antipsychotic drugs are at least partly metabolised by 2D6. Fluoxetine has been reported to increase plasma clozapine levels by around $80 \%$ (Centorrino et al, 1994); levels are known to be directly related to serious adverse effects such as seizures (Simpson \& Cooper, 1978). Case reports in the literature also seem to suggest pharmacokinetic potentiation of other antipsychotics such as fluphenazine (Ketai, 1993). Fluoxetine should therefore be used cautiously in patients taking antipsychotic medication and perhaps avoided in those taking clozapine. There is a dearth of reports of interactions involving other SSRIs, perhaps because they have been more recently introduced. Nevertheless, they too should be used carefully. In vitro data favour the use of citalopram where inhibition of p450-2D6 would be dangerous.

Some SSRIs may show clinically relevant inhibition of other cytochrome enzymes. Fluvoxamine inhibits theophylline metabolism by p450-1A2 in vitro (Rasmussen et al, 1995) and may cause substantial elevation of theophylline serum levels when the two drugs are given together (Thomson et al, 1992). This combination should be avoided. Cytochrome p450-1A2 is also responsible for caffeine metabolism (Slaughter \& Edwards, 1995) and so an increase in the adverse effects of caffeine might be expected in patients taking fluvoxamine. The importance of fluvoxamine's inhibition of $1 \mathrm{~A} 2$ in the reported interactions with tricyclics is not yet known but the drug does seem to inhibit more than one route of metabolism in vivo (Hartter et al, 1993).

Fluoxetine may inhibit cytochrome p450-3A3/4 (Preskorn \& Magnus, 1994) since it raises 
alprazolam plasma concentrations by $30 \%$ when the two drugs are given together (Lasher et al, 1991). Increased psychomotor impairment may result. In addition, two recent case reports seem to confirm fluoxetine's inhibitory effect on p450-3A4. Horton \& Bonser (1995) described substantial elevation of cyclosporin plasma levels soon after starting fluoxetine therapy. Administration of fluoxetine also appeared to be responsible for $\mathrm{QT}_{\mathrm{c}}$ abnormalities observed in a patient also taking terfenadine (Marchiando \& Cook, 1995).

Cimetidine and ketoconazole are strong inhibitors of p450 enzymes (Murray, 1992) and they are involved in a number of clinically relevant interactions with psychotropic drugs. However, their use is rarely necessary: ranitidine substitutes for cimetidine and fluconazole for ketoconazole. These alternatives are less likely to interact with psychotropic agents. Quinidine is also a potent inhibitor of p450-2D6 but is now rarely used clinically as there are a number of suitable alternatives (digoxin, disopyramide, amiodarone).

The cytochrome-mediated adverse drug interactions described here were, in general, discovered by chance and involved significant patient morbidity before discovery. As knowledge of the mechanisms of adverse interaction has advanced, in vitro methods have been developed and used to estimate the potential for interaction. Newer drugs (e.g. citalopram) have been tested for interactions with other psychotropics metabolised by p450 enzymes. In the future, adverse interactions may be predicted by computer modelling and molecular modelling techniques. This latter method has already been used to predict routes of metabolism mediated via p450-2D6 (Slaughter \& Edwards, 1995).

Thus, in vitro techniques can predict potential drug interactions involving cytochrome p450mediated metabolic pathways. This focuses clinical research activity towards direct evaluations of drug interactions in normal subjects and patients. The prescriber can then be provided with accurate information to improve the safety of patient care.

\section{References}

Alderman, J., Greenblatt, D. J., Allison, J., et al (1994) Desipramine pharmacokinetics with the serotonin reuptake inhibitors, paroxetine or sertraline. Data on File. Sandwich, Kent: Pfizer.

BARros, J. \& AsNis, G. (1993) An interaction of sertraline and desipramine. American Journal of Psychiatry, 150, 1751.

Brosen, K., SkJelbo, E., Rasmussen, B. B., et al (1993)

Fluvoxamine is a potent inhibitor of cytochrome P450-1A2. Biochemical Pharmacology, 45, 1211-1214.
Centorrino, F., Baldessarini, R.J., Kando, J., et al (1994) Serum concentrations of clozapine and its major metabolites: effects of co-treatment with fluoxetine or valproate. American Journal of Psychiatry, 151, 123-125.

Cholerton, S., Daly, A. K. \& IDle, J. R. (1992) The role of individual human cytochromes P450 in drug metabolism and clinical response. Trends in Pharmacological Science, 13, $434-439$.

Crewe, H. K., Lennard, M. S., Tucker, G. T., et al (1992) The effect of selective serotonin re-uptake inhibitors on cytochrome P450 2D6 (CYP2D6) activity in human liver microsomes. British Journal of Clinical Pharmacology, 34, 262-265.

Fischer, V., Vogels, B., Maurer, G., et al (1992) The antipsychotic clozapine is metabolised by the polymorphic human microsomal and recombinant cytochrome P450 2D6. Journal of Pharmacology and Experimental Therapeutics, 260, 1355-1360.

GAscon, M.-P. \& DAYER, P. (1991) In vitro forecasting of drugs which may interfere with the biotransformation of midazolam. European Journal of Clinical Pharmacology, 41, 573-578.

Gorski, J. C., HALl, S. D., JoNES, D. R., et al (1994) Regioselective biotransformation of midazolam by members of the human cytochrome P450 3A (CYP 3A) subfamily. Biochemical Pharmacology, 47, 1643-1653.

Gram, L. F., Hansen, M. G. J., Sindrup, S. H., et al (1993) Citalopram: Interaction studies with levomepromazine, imipramine, and lithium. Therapeutic Drug Monitoring, 15, 18-24.

Guengerich, F. P. (1992) Human cytochrome P-450 enzymes. Life Sciences, 50, 1471-1478.

HAhN, S. M. \& GRIfFIN, J. H. (1991) Comment: fluoxetine adverse effects of drug interactions. DICP - Annals of Pharmacotherapy, 25, 1273-1274.

Hartter, S. H., Wetzel, H., Hammes, E., et al (1993) Inhibition of antidepressant demethylation and hydroxylation by fluvoxamine in depressed patients. Psychopharmacology, 110, 302-308.

Horton, R. C. \& Bonser, R. S. (1995) Interaction between cyclosporin and fluoxetine. British Medical Journal, 311, 422.

KETAI, R. (1993) Interaction between fluoxetine and neuroleptics. American Journal of Psychiatry, 150, 836-837.

Kronbach, T., Mathys, D., Umeno, M., et al (1989) Oxidation of midazolam and triazolam by human liver cytochrome P450 IIIA4. Molecular Pharmacology, 36, 89-96.

Lasher, T. A., Fleishaker, J. C., Steenwiuk, R. C., et al (1991) Pharmacokinetic and pharmacodynamic evaluation of the combined administration of alprazolam and fluoxetine. Psychopharmacology, 104, 323-327.

Marchiando, R. J. \& Cook, M. D. (1995) Probable terfenadinefluoxetine-associated cardiac toxicity. Annals of Pharmacotherapy, 29, 937-938.

MASKALL, D. D. \& LAM, R. W. (1993) Increased plasma concentration of imipramine following augmentation with fluvoxamine. American Journal of Psychiatry, 150, 1566.

Murray, M. (1992) P450 enzymes: inhibition mechanisms, genetic regulation and effects of liver disease. Clinical Pharmacokinetics, 23, 132-146.

Preskorn, S. H., Beber, J. H., Faul, J. C., et al (1990) Serious adverse effects of combining fluoxetine and tricyclic antidepressants. American Journal of Psychiatry, 147, 532. -, Alderman, J., Chung, M., et al (1994) Pharmacokinetics of desipramine co-administered with sertraline or fluoxetine. Journal of Clinical Psychopharmacology, 14, 90-98.

- \& MAgNuS, R. D. (1994) Inhibition of hepatic P-450 isoenzymes by selective serotonin reuptake inhibitors: In vitro and in vivo findings and their implications for patient care. Psychopharmacology Bulletin, 30, 251-259.

Rasmussen, B. B., MaenpaA, J., Pelkonen, O., et al (1995) Selective serotonin reuptake inhibitors and theophylline metabolism in 
human liver microsomes: potent inhibition by fluvoxamine. British Journal of Clinical Pharmacology, 39, 151-159.

Simpson, G. M. \& CoOper, T. A. (1978) Clozapine plasma levels and convulsions. American Journal of Psychiatry, 135, 99-100. Slaughter, R. L. \& Edwards, D. J. (1995) Recent advances: The cytochrome P450 enzymes. Annals of Pharmacotherapy, 29, 619-624.

Thomson, A. H., McGovern, E. M., Bennie, P., et al (1992) Interaction between fluvoxamine and theophylline. Pharma ceutical Journal, 249, 137. von Moltke, L. L., Greenblatt, D. J., Harmatz, J. S., et al (1993) Alprazolam metabolism in vitro: studies of human, monkey, mouse, and rat liver microsomes. Pharmacology, 47, 268-276

- — - et al (1994) Cytochromes in psychopharmacology. Journal of Clinical Psychopharmacology, 14, 1-2.

Watkins, P. B. (1990) Role of cytochromes P450 in drug metabolism and hepatotoxicity. Seminars in Liver Disease, 10, 235-250.

David Taylor, MSc, Malcolm Lader, DSc, Maudsley Hospital, London SE5 8AF

Correspondence: Professor M. Lader, Maudsley Hospital, Denmark Hill, London SE5 8AF. Fax: 01719192171

(First received 6 September 1995, final revision 18 October 1995, accepted 17 November 1995) 